\title{
Differential expression of ELMO domain-containing 3 in cancers of the breast.
}

Shahan Mamoor, MS ${ }^{1}$

$3 \quad{ }^{1}$ shahanmamoor@gmail.com

East Islip, NY 11730

Breast cancer affects women at relatively high frequency ${ }^{1}$. We mined published microarray datasets ${ }^{2,3}$ to determine in an unbiased fashion and at the systems level genes most differentially expressed in the primary tumors of patients with breast cancer. We report here significant differential expression of the gene encoding ELMO domain-containing 3, ELMOD3, when comparing primary tumors of the breast to the tissue of origin, the normal breast. ELMOD3 was also differentially expressed in the brain metastases of patients with metastatic breast cancer. ELMOD3 mRNA was present at significantly lower quantities in tumors of the breast as compared to normal breast tissue. Analysis of human survival data revealed that expression of ELMOD3 in primary tumors of the breast was correlated with recurrence-free survival in patients with basal, luminal A, and luminal B subtype cancer, demonstrating a relationship between primary tumor expression of a differentially expressed gene and patient survival outcomes influenced by PAM50 molecular subtype. ELMOD3 may be of relevance to initiation, maintenance or progression of cancers of the female breast.

Keywords: breast cancer, ELMOD3, ELMO domain-containing 3, systems biology of breast cancer, targeted therapeutics in breast cancer. 
Invasive breast cancer is diagnosed in over a quarter of a million women in the United States each year $^{1}$ and in 2018 , breast cancer was the leading cause of cancer death in women worldwide ${ }^{4}$. While patients with localized breast cancer are provided a 99\% 5-year survival rate, patients with regional breast cancer, cancer that has spread to lymph nodes or nearby structures, are provided an $86 \% 5$-year survival rate $^{5,6}$. Patients with metastasis to distant sites, like the brain, are provided a $27 \% 5$-year survival rate ${ }^{5,6}$. Understanding how primary tumors are most transcriptionally different from the tissue from which they originate, the breast, can facilitate development of novel diagnostic and therapeutics to promote early detection and enhanced treatment, and contribute to efforts to prevent progression to metastatic stages. We mined published microarray data ${ }^{2,3}$ to understand at the transcriptome level and in an unbiased fashion genes most differentially expressed in primary tumors of the breast as compared to normal breast tissue. ELMO domain-containing 3 emerged as among the most differentially expressed genes in cancer of the female breast.

\section{Methods}

We utilized datasets GSE42568 ${ }^{2}$ and GSE52604 ${ }^{3}$ for this global differential gene expression analysis of female breast cancer. GSE42568 was generated using Affymetrix Human Genome U133 Plus 2.0 array technology with $n=17$ normal breast tissue biopsies and $n=104$ primary breast tumor biopsies from patients with breast cancer; analysis was performed using platform GPL570; the majority of patients whose tumors were analyzed were age 50 or older. GSE52604 was generated using Agilent-014850 Whole Human Genome Microarray 4x44K G4112F with $n=10$ normal breast tissues and $n=35$ brain metastases from patients with breast cancer; analysis was performed using platform GPL6480. The Benjamini and Hochberg method of $p$-value adjustment was used for ranking of differential expression but raw $p$-values were used to assess statistical significance of global differential expression.

Log-transformation of data was auto-detected, and the NCBI generated category of platform annotation was used. A statistical test was performed to evaluate whether ELMOD3 expression was significantly different between primary breast tumors and normal breast tissue using a two-tailed t-test. For Kaplan-Meier survival analysis, we used the Kaplan-Meier plotter online tool ${ }^{7}$ for correlation of ELMOD3 mRNA expression levels with recurrence-free survival (RFS) in $n=442$ patients with basal-like subtype cancer, $n=631$ patients with luminal A subtype cancer, $n=566$ patients with luminal B subtype cancer, $n=358$ patients with HER $2+$ cancer, and $n=35$ patients with normal-like subtype cancer.

\section{Results}

We performed discovery of genes associated with breast cancer in females by mining two independently published microarray datasets ${ }^{2,3}$.

\section{ELMOD3 is differentially expressed in primary tumors of the breast.}

Studying the global gene expression profiles of 104 breast cancers from patients aged 31 to 89 revealed that the gene encoding ELMO domain-containing 3, ELMOD3, was among the genes most differentially expressed in tumors of the breast in human breast cancer (Chart 1). When sorting each of the genes expressed in tumors of the breast based on significance of difference as compared to normal breast tissue, ELMOD3 ranked 127 out of 54675 total transcripts, equating to $99.8 \%$ differential expression (Chart 1). Differential expression of ELMOD3 in female breast cancer was statistically significant (Chart $1 ; p=1.85 \mathrm{E}-29)$.

Analysis of a second microarray dataset ${ }^{3}$, here studying global gene expression patterns in the brain metastases of patients with metastatic breast cancer, again revealed significant differential expression of ELMOD3 in tumor tissue of patients with human breast cancer (Chart 2). When sorting each of the genes 
expressed in the brain metastases of patients with breast cancer based on significance of difference as compared to normal breast tissue, ELMOD3 ranked 12776 out of 41093 total transcripts, equating to $68.9 \%$ differential expression (Chart 2). Differential expression of ELMOD3 in the brain metastases of patients with breast cancer was statistically significant (Chart $2 ; p=1.46 \mathrm{E}-02$ ). These data suggested that differential expression of ELMOD3 was not an artifact of a single microarray dataset, nor was it strictly associated with primary tumors of the breast, rather a reflection of the spectrum of transformation in human cancer.

\section{ELMOD3 is expressed at significantly lower levels in breast tumors as compared to the breast.}

We obtained exact mRNA expression levels for ELMOD3 from the breast and from breast tumors to understand the magnitude and direction of ELMOD3 expression change. ELMOD3 was expressed at lower levels in tumors of the breast as compared to normal breast tissue (Figure 1). Decreased expression of ELMOD3 in primary breast tumors was statistically significant (Figure $1: p<0.0001$ ). ELMOD3 was expressed at $6.12 \pm 1.60$ arbitrary units (AU) in normal breast tissue, while it was expressed at $3.62 \pm$ $0.27 \mathrm{AU}$ in tumors of the breast. We calculated a mean fold change of 0.59 in ELMOD3 mRNA levels when comparing primary tumors of the breast to normal breast tissues.

\section{ELMOD3 expression correlates with survival outcomes in basal-like, luminal A, and luminal B} subtype human breast cancer.

We performed Kaplan-Meier survival analysis to study relationships between tumor ELMOD3 mRNA expression levels and survival outcomes in patients with breast cancer.

We observed a correlation between ELMOD3 expression and recurrence-free survival (RFS) in patients with basal-like subtype breast cancer which was statistically significant (Figure 2; log rank $p$-value: 0.0031 for recurrence-free survival, hazard ratio: 0.63 (0.46-0.86) (Fig. 2)). ELMOD3 mRNA levels were a positive prognostic indicator in basal-like subtype breast cancer patients. Median RFS was 17 months for basal-like patients with low tumor expression of ELMOD3 while median RFS was 30.42 months for basal-like patients with high tumor expression of ELMOD3 (Chart 3).

We also observed a correlation between ELMOD3 expression and recurrence-free survival (RFS) in patients with luminal A subtype breast cancer which was statistically significant (Figure 2; $\log$ rank $p$-value: 0.00025 for recurrence-free survival, hazard ratio: 0.52 (0.36-0.74) (Fig. 2)). ELMOD3 mRNA levels were a positive prognostic indicator in luminal A subtype breast cancer patients. RFS was, on average, markedly shorter for luminal A patients with low tumor expression of ELMOD3 as compared to luminal A patients with high tumor expression of ELMOD3 (Chart 3).

We also observed a correlation between ELMOD3 expression and recurrence-free survival (RFS) in patients with luminal B subtype breast cancer which trended towards statistical significance (Figure 2; $\log$ rank $p$-value: 0.076 for recurrence-free survival, hazard ratio: 0.79 (0.61-1.03) (Fig. 2)). ELMOD3 mRNA levels were a positive prognostic indicator in luminal B subtype breast cancer patients. Median RFS was 31 months for luminal B patients with low tumor expression of ELMOD3 while median RFS was 39 months for luminal B patients with high tumor expression of ELMOD3 (Chart 3). ELMOD3 primary tumor expression was not correlated with recurrence-free survival in HER2+ breast cancer (Figure 2; log rank $p$-value: 0.1 for RFS, hazard ratio: $0.76(0.55-1.06)$ (Fig. 2)) or in patients with normal-like breast cancer (Figure 2; log rank $p$-value: 1 for RFS, hazard ratio: 1 (0.38-2.68) (Fig. 2)).

Thus, through comparative transcriptome analysis of primary tumors of the breast and normal breast tissue, we found that differential expression and down-regulation of ELMOD3 was among the most significant transcriptional features in primary tumors from patients with breast cancer. ELMOD3 expression in primary tumors of the breast was correlated with recurrence-free survival in patients with luminal A, luminal B, and basal-like subtype disease, with mRNA levels of ELMOD3 a positive 
prognostic indicator for basal-like, luminal A and luminal B breast cancer patients.

\section{Discussion}

Invasive breast cancer is a medical problem with a $27 \% 5$-year survival rate for women whose disease has spread to distant sites ${ }^{5,6}$. To facilitate understanding of the basic transcriptional differences between primary tumors of the breast and the tissues from which these tumors originate, normal breast tissues, we performed comparative transcriptome analysis using two independently published microarray datasets $^{2,3}$, providing evidence here that differential expression of ELMO domain-containing 3, encoded by ELMOD3, is a defining transcriptional feature of human breast cancer: in patients diagnosed after age 50, and in brain metastatic tissues from patients with human breast cancer. ELMOD3 was expressed at significantly lower levels in primary tumors from patients with breast cancer as compared to normal breast tissue. Importantly, in patients with basal-like, luminal A and luminal B subtype breast cancers, expression of ELMOD3 was correlated with recurrence-free survival. ELMO domain-containing 3 and breast cancer. 


\section{References}

1. DeSantis, C.E., Ma, J., Goding Sauer, A., Newman, L.A. and Jemal, A., 2017. Breast cancer statistics, 2017, racial disparity in mortality by state. CA: a cancer journal for clinicians, 67(6), pp.439-448.

2. Clarke, C., Madden, S.F., Doolan, P., Aherne, S.T., Joyce, H., O’driscoll, L., Gallagher, W.M., Hennessy, B.T., Moriarty, M., Crown, J. and Kennedy, S., 2013. Correlating transcriptional networks to breast cancer survival: a large-scale coexpression analysis. Carcinogenesis, 34(10), pp.2300-2308.

3. Salhia, B., Kiefer, J., Ross, J.T., Metapally, R., Martinez, R.A., Johnson, K.N., DiPerna, D.M., Paquette, K.M., Jung, S., Nasser, S. and Wallstrom, G., 2014. Integrated genomic and epigenomic analysis of breast cancer brain metastasis. Plos one, $9(1)$, p.e85448.

4. Bray, F., Ferlay, J., Soerjomataram, I., Siegel, R.L., Torre, L.A. and Jemal, A., 2018. Global cancer statistics 2018: GLOBOCAN estimates of incidence and mortality worldwide for 36 cancers in 185 countries. CA: a cancer journal for clinicians, 68(6), pp.394-424.

5. ACS Cancer Facts \& Figures 2019.

https://www.cancer.net/cancer-types/breast-cancermetastatic/statistics.

6. Survival Rates for Breast Cancer. https://www.cancer.org/cancer/breast-cancer/ understanding-a-breast-cancer-diagnosis/breast-cancer-survival-rates.html

7. Györffy, B., Lanczky, A., Eklund, A.C., Denkert, C., Budczies, J., Li, Q. and Szallasi, Z., 2010. An online survival analysis tool to rapidly assess the effect of 22,277 genes on breast cancer prognosis using microarray data of 1,809 patients. Breast cancer research and treatment, 123(3), pp.725-731. 


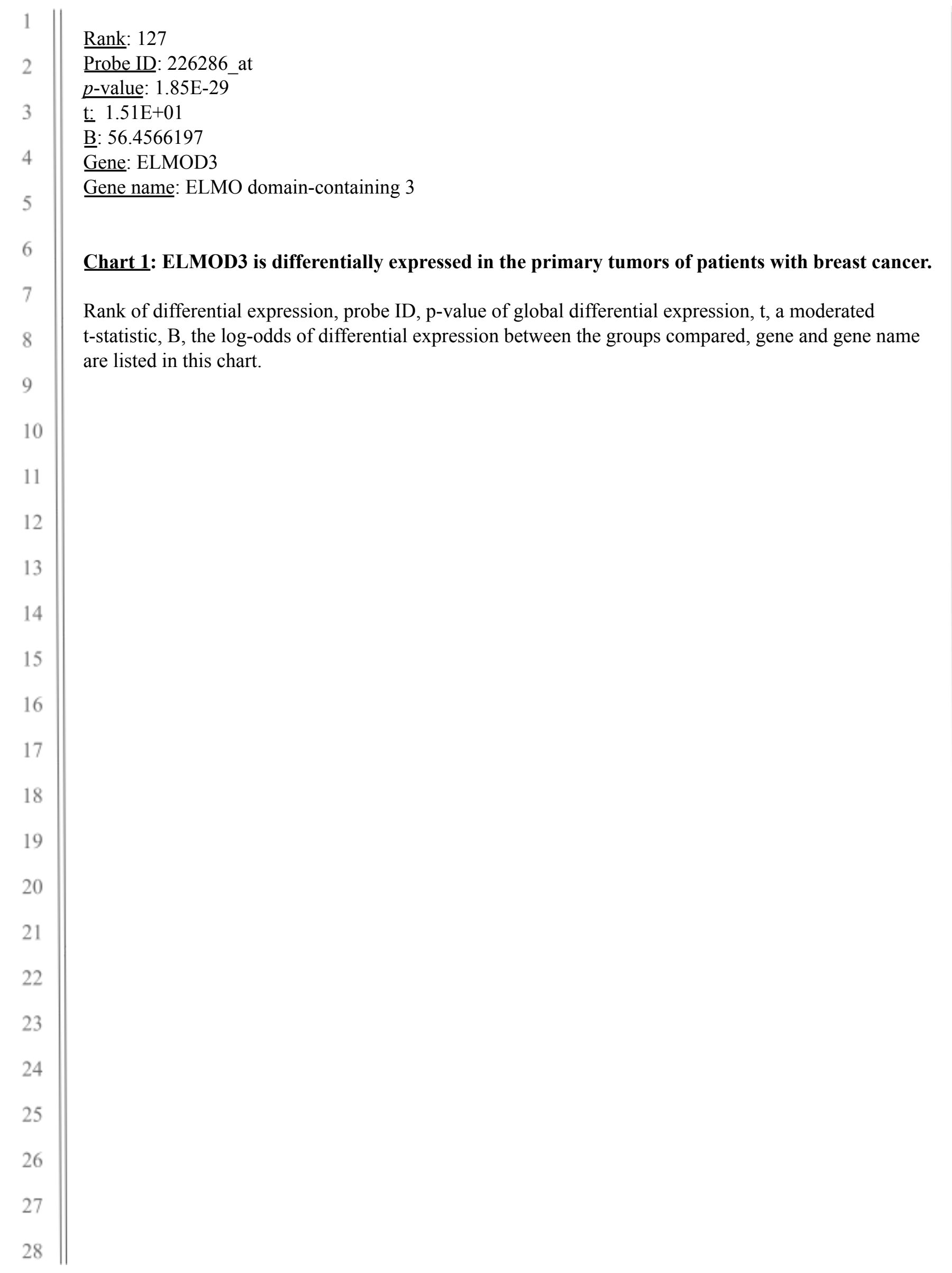




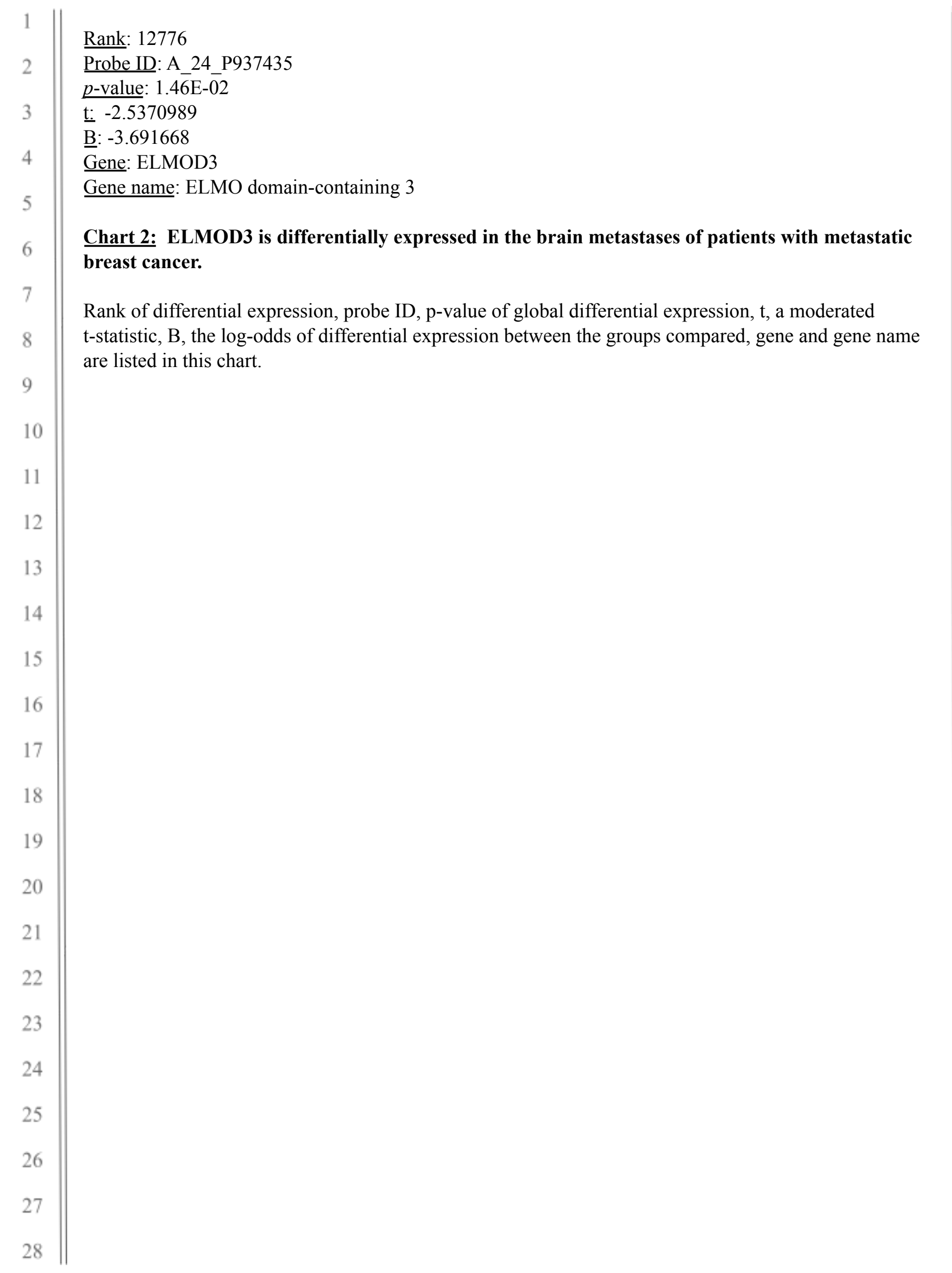




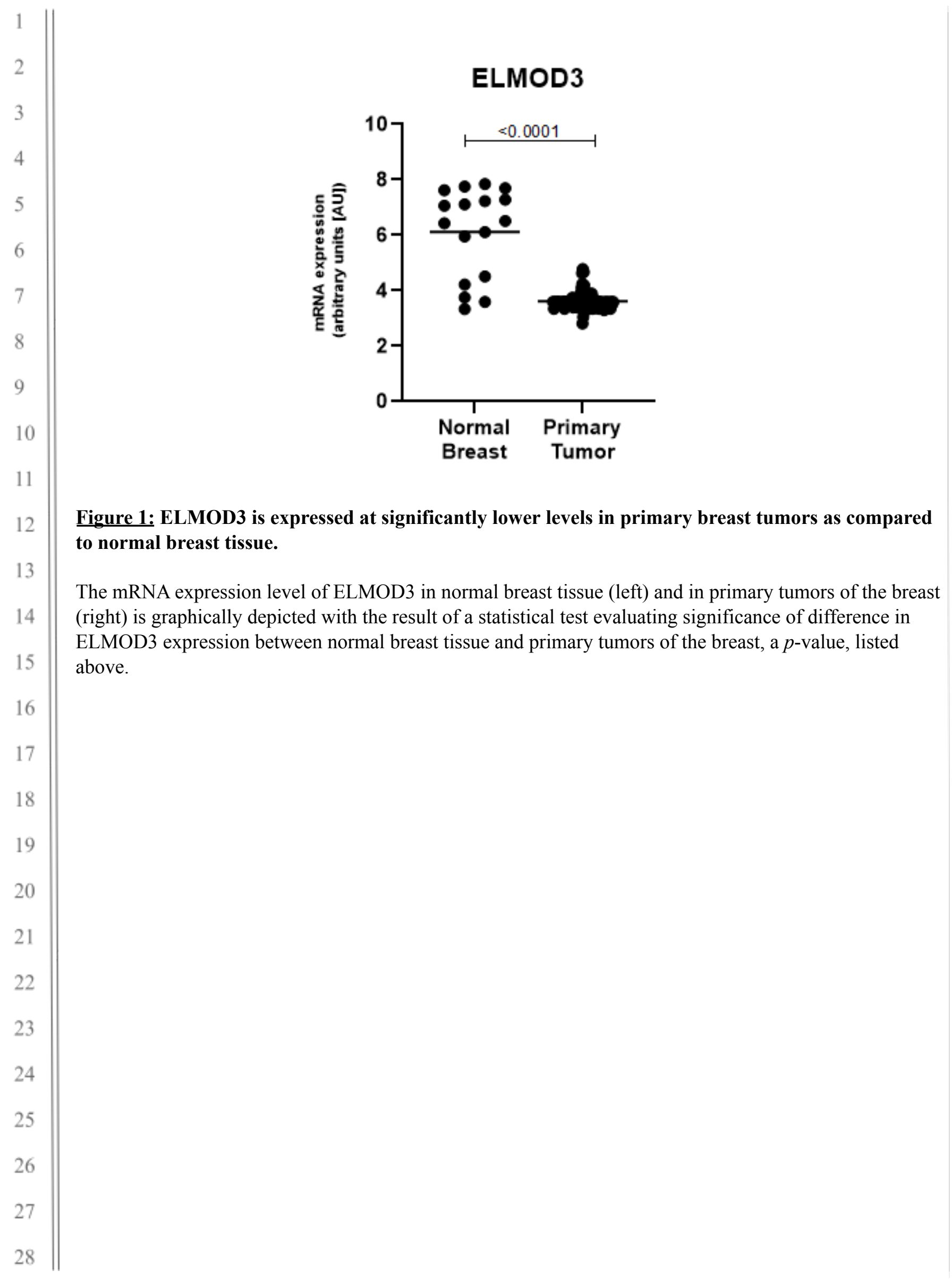




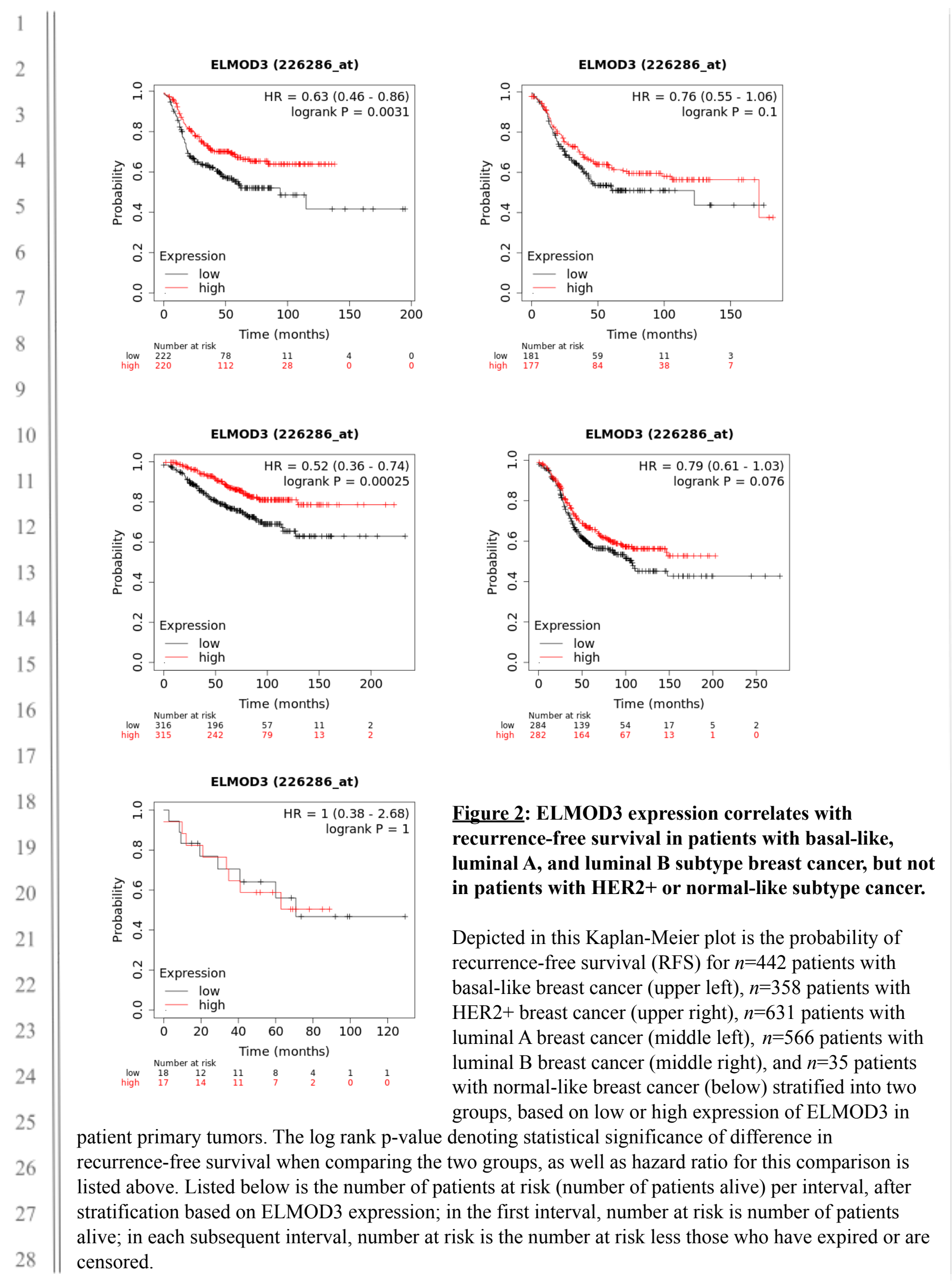




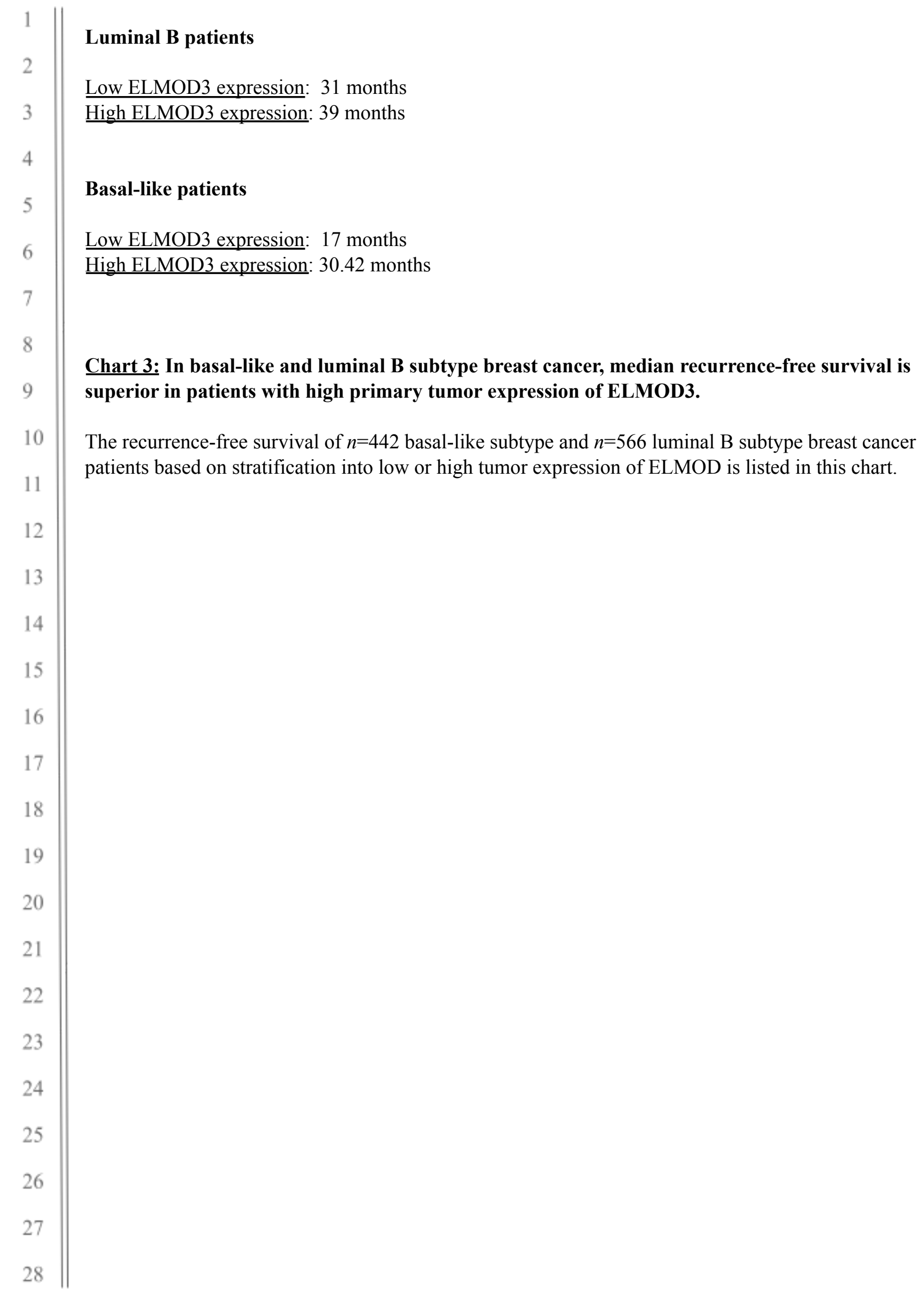

